\title{
STABILITY OF INVARIANT SETS OF ITÔ STOCHASTIC DIFFERENTIAL EQUATIONS WITH MARKOVIAN SWITCHING
}

\author{
JIAOWAN LUO
}

Received 16 January 2004; Revised 26 September 2005; Accepted 26 September 2005

Consider the nonlinear Itô stochastic differential equations with Markovian switching, some sufficient conditions for the invariance, stochastic stability, stochastic asymptotic stability, and instability of invariant sets of the equations are derived.

Copyright (c) 2006 Jiaowan Luo. This is an open access article distributed under the Creative Commons Attribution License, which permits unrestricted use, distribution, and reproduction in any medium, provided the original work is properly cited.

\section{Introduction}

Invariant sets of dynamic systems play an important role in many situations when the dynamic behavior is constrained in some way. Knowing that a set in the state space of a system is invariant means that we have bounds on the behavior. We can verify that pre-specified bounds which originate from, for example, safety restrictions, physical constraints, or state-feedback magnitude bounds are not invalidated.

There is significant literature devoted to the invariant sets of ordinary differential equations, functional differential equations, and stochastic differential equations, and we here mention [2, 4, 15-18].

Recently, much work has been done on stochastic differential equations with Markovian switching $[1,3,5-14,19,20]$. In particular, we here highlight Mao's significant contribution $[6,11,12]$. However, to the best of the author's knowledge to date, the problem of the invariant sets of equations of this kind, has not been investigated yet.

The aim of the present paper is to study the invariant sets of nonlinear Itô stochastic differential equations with Markovian switching. Similar to the result of [18], which investigates the usual stochastic differential equations, some sufficient conditions for the invariance and stochastic stability of invariant sets of equations of this kind are derived. At the same time, we establish some conditions for stochastic asymptotic stability and instability of the invariant sets, which are not discussed in [18] even in the case of equations without Markovian switching. 
2 Invariant sets of stochastic equations with Markovian switching

\section{Stochastic differential equations with Markovian switching}

Let $\left\{\Omega, \mathscr{F}_{F},\left\{\mathscr{F}_{t}\right\}_{t \geq 0}, P\right\}$ be a complete probability space with a filtration satisfying the usual conditions, that is, the filtration is continuous on the right and $\mathscr{F}_{0}$ contains all $P$-zero sets. Let $w(t)=\left(w_{1}(t), w_{2}(t), \ldots, w_{m}(t)\right)^{T}$ be an $m$-dimensional Brownian motion defined on the probability space. Let $|\cdot|$ is the Euclidean norm in $R^{n}$, that is, $|x|=\sqrt{x^{T} x}\left(x \in R^{n}\right)$.

Let $\left\{r(t), t \in R_{+}=[0,+\infty]\right\}$ be a right-continuous Markov chain on the probability space $\left\{\Omega, \mathscr{F},\left\{\mathscr{F}_{t}\right\}_{t \geq 0}, P\right\}$ taking values in a finite state space $S=\{1,2, \ldots, N\}$ with generator $\Gamma=\left(\gamma_{i j}\right)_{N \times N}$ given by

$$
P\{r(t+\delta)=j \mid r(t)=i\}= \begin{cases}\gamma_{i j} \delta+o(\delta), & \text { if } i \neq j \\ 1+\gamma_{i i} \delta+o(\delta), & \text { if } i=j\end{cases}
$$

where $\delta>0$. Here $\gamma_{i j} \geq 0$ is the transition rate from $i$ to $j$ if $i \neq j$, while $\gamma_{i i}=-\sum_{j \neq i} \gamma_{i j}$. We assume that the Markov chain $r(\cdot)$ is independent of the Brownian motion $w(\cdot)$. It is known that almost every sample path of $r(t)$ is a right-continuous step function with a finite number of simple jumps in any finite subinterval of $R_{+}$, and $r(t)$ is ergodic.

Consider the Itô stochastic differential equations with Markovian switching:

$$
d x(t)=f(t, x(t), r(t)) d t+g(t, x(t), r(t)) d w(t),
$$

where $t \geq 0, f: R_{+} \times R^{n} \times S \rightarrow R^{n}, g: R_{+} \times R^{n} \times S \rightarrow R^{n \times m}$, and the initial condition is $x\left(t_{0}\right)=x_{0} \in R^{n}, r\left(t_{0}\right)=r_{0} \in S, t_{0} \geq 0$.

In this paper we always assume that both $f$ and $g$ satisfy the local Lipschitz condition and the linear growth condition. Hence it is known from [6] that (2.2) has a unique continuous bounded solution $x(t)=x\left(t, t_{0}, x_{0}\right)$ on $t \geq t_{0}$.

Denote by $C^{2,1}\left(R_{+} \times R^{n} \times S ; R_{+}\right)$the family of all nonnegative functions $V(t, x, i)$ on $R_{+} \times R^{n} \times S$ which are continuously twice differentiable with respect to $x$ and once differentiable with respect to $t$. For any $(t, x, i) \in R_{+} \times R^{n} \times S$, we define an operator $\mathscr{L}$ by

$$
\begin{aligned}
\mathscr{L} V(t, x, i)= & \sum_{j=1}^{N} \gamma_{i j} V(t, x, j)+V_{t}(t, x, i)+V_{x}(t, x, i) f(t, x, i) \\
& +\frac{1}{2} \operatorname{trace}\left[g^{T}(t, x, i) V_{x x}(t, x, i) g(t, x, i)\right],
\end{aligned}
$$

where

$$
\begin{gathered}
V_{t}(t, x, i)=\frac{\partial V(t, x, i)}{\partial t}, \quad V_{x}(t, x, i)=\left(\frac{\partial V(t, x, i)}{\partial x_{1}}, \ldots, \frac{\partial V(t, x, i)}{\partial x_{n}}\right), \\
V_{x x}(t, x, i)=\left(\frac{\partial^{2} V(t, x, i)}{\partial x_{i} \partial x_{j}}\right)_{n \times n} .
\end{gathered}
$$

The generalized Itô formula reads as follows: if $V \in C^{2,1}\left([-\tau,+\infty) \times R^{n} \times S ; R_{+}\right)$, then

$$
E V(t+h, x(t+h), r(t+h))=E V(t, x(t), r(t))+E \int_{t}^{t+h} \mathscr{L} V(s, x(s), r(s)) d s .
$$




\section{Main results}

Denote by $Q$ a certain Borel set in $\{t \geq 0\} \times R^{n} \times S$. Let $Q_{t}^{r(t)}$ be a set in $R^{n}$, here $Q_{t}^{r(t)}=$ $\{x(t):(t, x(t), r(t)) \in Q\}$ and $Q_{t}^{r(t)}$ is nonempty for $t \geq 0$.

Definition 3.1. A set $Q$ is called invariant for (2.2) if for $\left(t_{0}, x_{0}, r_{0}\right) \in Q$,

$$
P\left\{(t, x(t), r(t)) \in Q, \forall t \geq t_{0}\right\}=1 .
$$

Remark 3.2. Definition 3.1 is equivalent to the condition

$$
P\{(t, x(t), r(t)) \in Q\}=1, \quad \forall t \geq t_{0} .
$$

Definition 3.3. A set $Q$ is called stochastically stable if for any $\varepsilon>0$ the following holds:

$$
\lim _{\rho\left(x_{0}, Q_{t_{0}}^{r_{0}}\right) \rightarrow 0} P\left\{\sup _{t \geq t_{0}} \rho\left(x(t), Q_{t}^{r(t)}\right)>\varepsilon\right\}=0,
$$

where $\rho(x, A)$ denotes the distance between a point $x$ and a set $A$.

Definition 3.4. A set $Q$ is called stochastically asymptotically stable if it is stochastically stable and moreover

$$
\lim _{\rho\left(x_{0}, Q_{t_{0}}^{r_{0}}\right) \rightarrow 0} P\left\{\lim _{t \rightarrow \infty} \rho\left(x(t), Q_{t}^{r(t)}\right)=0\right\}=1 .
$$

Definition 3.5. A set $Q$ is called unstable if there exist $\varepsilon_{1}>0$ and $\varepsilon_{2}>0$ such that, for any $\delta>0$, there exist $x_{0}$ and $t^{*}$ such that for $\rho\left(x_{0}, Q_{t_{0}}^{r_{0}}\right)<\delta$ the following holds:

$$
P\left\{\sup _{t^{*} \geq t \geq t_{0}} \rho\left(x, Q_{t}^{r(t)}\right)>\varepsilon_{1}\right\} \geq \varepsilon_{2} .
$$

Denote by $\Gamma$ the set of its zeros of the function $V$ in $\{t \geq 0\} \times R^{n} \times S$, that is, $\Gamma=$ $\{(t, x, i): V(t, x, i)=0\}$. Let $\Gamma_{t}^{i}$ denote the set of $x \in R^{n}$ such that $V(t, x, i)=0$ for fixed $t \geq 0$ and $i \in S$.

THeOREM 3.6. If

$$
\mathscr{L} V(t, x, i) \leq 0,
$$

then the set $\Gamma$ is a positive invariant set for (2.2). In addition, if

$$
\inf _{\rho\left(x, \Gamma_{t}^{i}\right)>\delta} V(t, x, i)=V_{\delta}>0
$$

for any $\delta>0$, then the set $\Gamma$ is stochastically stable. 
4 Invariant sets of stochastic equations with Markovian switching

Proof. Without loss of generality, we assume that $t_{0}=0$, that is, $x\left(0, x_{0}\right)=x_{0} \in \Gamma_{0}^{i}$. Applying (2.5) we have

$$
E V(t, x(t), i)=E V\left(0, x_{0}, i\right)+E \int_{0}^{t} \mathscr{L} V(s, x(s), i) d s
$$

From (3.6), we get

$$
E V(t, x, i) \leq 0
$$

that is,

$$
V(t, x, i)=0 \quad \text { a.e. }
$$

Thus $\Gamma$ is invariant.

Assume that $\varepsilon_{1}$ and $\varepsilon_{2}$ are arbitrary positive constants. Denote $\inf _{\rho\left(x, \Gamma_{t}^{i}\right)>\varepsilon_{1}} V(t, x, i)=$ $V_{\varepsilon_{1}}$. Then, from (3.7) we know that $V_{\varepsilon_{1}}>0$. It is easily seen that

$$
P\left\{\sup _{t \geq t_{0}} \rho\left(x, \Gamma_{t}^{i}\right)>\varepsilon_{1}\right\} V_{\varepsilon_{1}} \leq E V(t, x, i) \leq V\left(t_{0}, x_{0}, i\right) .
$$

By the right-continuity of the function $V$, there exists $\delta>0$ for $\varepsilon_{2}>0$ such that for $\rho\left(x_{0}, \Gamma_{t_{0}}^{r_{0}}\right)<\delta$, we get

$$
V\left(t_{0}, x_{0}, i\right) \leq V_{\varepsilon_{1}} \varepsilon_{2}
$$

By use of (3.11) and (3.12), we complete the proof.

Remark 3.7. It is obvious that the condition (3.7) is automatically satisfied if the function $V$ is independent on $t$.

Remark 3.8. If both of the coefficients of (2.2), $f$ and $g$, are independent of the Markov chain $r(t)$, then Theorem 3.6 in this paper reduces to [18, Theorem 1].

Theorem 3.9. Suppose that (3.7) and the following hold:

$$
\mathscr{L} V(t, x, i) \leq-\varphi(V(t, x, i))
$$

where $\varphi(s), s \geq 0$ is a continuous function, $\varphi(0)=0, \varphi(s)>0$ for $s>0$, and $E \varphi(\tau) \geq \varphi(E \tau)$ for every nonnegative random variable $\tau$. Then the set $\Gamma$ is a stochastically asymptotically stable invariant set for (2.2).

Proof. Since the conditions of Theorem 3.6 are satisfied, $\Gamma$ is a stable invariant set. Hence, for any sufficiently small $\varepsilon_{1}>0$ and $\varepsilon_{2}>0$, there exists $\delta>0$ such that for $\rho\left(x_{0}, \Gamma_{t_{0}}^{i}\right)<\delta$, we have $P\left\{\sup _{t \geq t_{0}} \rho\left(x(t), \Gamma_{t}^{i}\right)>\varepsilon_{1}\right\}<\varepsilon_{2}$.

It is obviously seen from (2.5) and (3.16) that the function $E V(t, x(t), i)$ is nonnegative and nonincreasing. Let $\alpha=\lim _{t \rightarrow+\infty} E V(t, x(t), i)$. Hence, $\alpha \geq 0$. Assume that $\alpha>0$. Then the inequality $\alpha \leq E V(t, x(t), i) \leq V_{\varepsilon_{1}}$ is true. Denote $c=\min _{\alpha \leq s \leq V_{\varepsilon_{1}}} \varphi(s)$. From (2.5) and (3.16), we get

$$
E V(t, x(t), i) \leq E V\left(0, x_{0}, i\right)-c t
$$


which, for sufficiently large $t$, contradicts the fact $\alpha>0$. Hence, we have $\alpha=0$. Thus, for $\rho\left(x_{0}, \Gamma_{t_{0}}^{i}\right)<\delta$, we get

$$
\lim _{t \rightarrow \infty} \rho\left(x(t), \Gamma_{t}^{i}\right)=0 \quad \text { a.e. }
$$

The proof is complete.

Theorem 3.10. Suppose that (3.7) and the following hold:

$$
\mathscr{L} V(t, x, i) \geq \varphi(V(t, x, i))
$$

where $\varphi(s), s \geq 0$ is a continuous function, $\varphi(0)=0, \varphi(s)>0$ for $s>0$, and $E \varphi(\tau) \leq \varphi(E \tau)$ for every nonnegative random variable $\tau$. Then the set $\Gamma$ is an unstable set for (2.2).

Proof. Let $\delta>0$ be a sufficiently small number. We choose $x_{0}$ such that $\rho\left(x_{0}, \Gamma_{t_{0}}^{i}\right)<\delta$ and assume that $V\left(0, x_{0}, i\right)=\alpha>0$. To the contrary, we assume that $\rho\left(x(t), \Gamma_{t}^{i}\right)<\varepsilon$ for $t>0$. From (2.5) and (3.16), it is seen that $E V(t, x, i)>0$. Thus there exists an $L$ such that $0<\alpha \leq E V(t, x, i) \leq L$. Denote $c=\min _{\alpha \leq s \leq L} \varphi(s)$. Equations (3.16) and (2.5) yield $E V(t, x, i) \geq V\left(0, x_{0}, i\right)+c t$, which contradicts the fact that $E V(t, x, i)$ is bounded. Thus, the assumption $\rho\left(x(t), \Gamma_{t}^{i}\right)<\varepsilon$ for $t>0$ is not true and the set $\Gamma$ is unstable.

\section{Acknowledgment}

This work was partially supported by NNSF of China (Grant no. 10301036).

\section{References}

[1] G. K. Basak, A. Bisi, and M. K. Ghosh, Stability of a random diffusion with linear drift, Journal of Mathematical Analysis and Applications 202 (1996), no. 2, 604-622.

[2] P. W. Bates, K. Lu, and C. Zeng, Existence and persistence of invariant manifolds for semiflows in Banach space, Memoirs of the American Mathematical Society 135 (1998), no. 645, 1-129.

[3] Y. Ji and H. J. Chizeck, Controllability, stabilizability, and continuous-time Markovian jump linear quadratic control, IEEE Transactions on Automatic Control 35 (1990), no. 7, 777-788.

[4] G. L. Kulīnīch and O. V. Pereguda, Qualitative analysis of systems of Itô stochastic differential equations, Ukrainian Mathematical Journal 52 (2000), no. 9, 1432-1438.

[5] J. Luo, J. Zou, and Z. Hou, Comparison principle and stability criteria for stochastic differential delay equations with Markovian switching, Science in China. Series A: Mathematics 46 (2003), no. $1,129-138$.

[6] X. Mao, Stability of stochastic differential equations with Markovian switching, Stochastic Processes and Their Applications 79 (1999), no. 1, 45-67.

[7] __ Stochastic functional differential equations with Markovian switching, Functional Differential Equations 6 (1999), no. 3-4, 375-396.

[8] __ Robustness of stability of stochastic differential delay equations with Markovian switching, Stability and Control: Theory and Applications 3 (2000), no. 1, 48-61.

[9] ___ Asymptotic stability for stochastic differential delay equations with Markovian switching, Functional Differential Equations 9 (2002), no. 1-2, 201-220.

[10] _ Asymptotic stability for stochastic differential equations with Markovian switching, WSEAS Transactions on Circuits 1 (2002), no. 1, 68-73.

[11] _ Exponential stability of stochastic delay interval systems with Markovian switching, IEEE Transactions on Automatic Control 47 (2002), no. 10, 1604-1612. 
[12] X. Mao, A. Matasov, and A. B. Piunovskiy, Stochastic differential delay equations with Markovian switching, Bernoulli 6 (2000), no. 1, 73-90.

[13] X. Mao and L. Shaikhet, Delay-dependent stability criteria for stochastic differential delay equations with Markovian switching, Stability and Control: Theory and Applications 3 (2000), no. 2, $88-102$.

[14] M. Mariton, Jump Linear Systems in Automatic Control, Marcel Dekker, New York, 1990.

[15] K. Sawano, Positively invariant sets for functional differential equations with infinite delay, Tôhoku Mathematical Journal. Second Series 32 (1980), no. 4, 557-566.

[16] G. Seifert, Positively invariant closed sets for systems of delay differential equations, Journal of Differential Equations 22 (1976), no. 2, 292-304.

[17] O. M. Stanzhits'ki1, Investigation of invariant sets with random perturbations by means of Lyapunov functions, Ukrainian Mathematical Journal 50 (1998), no. 2, 355-359.

[18] - Investigation of invariant sets of Itô stochastic systems by means of Lyapunov functions, Ukrainian Mathematical Journal 53 (2001), no. 2, 323-327.

[19] A. V. Svīshchuk and Yu. İ. Kazmerchuk, Stability of stochastic Itô equations with delays, with jumps and Markov switchings, and their application in finance, Theory of Probability and Mathematical Statistics (2002), no. 64, 167-178.

[20] C. Yuan and X. Mao, Asymptotic stability in distribution of stochastic differential equations with Markovian switching, Stochastic Processes and Their Applications 103 (2003), no. 2, 277-291.

Jiaowan Luo: Department of Probability and Statistics, School of Mathematics and Information Sciences, Guangzhou University, Guangzhou, Guangdong 510006, China E-mail addresses: jwluo@mail.csu.edu.cn; mathluo@yahoo.com 


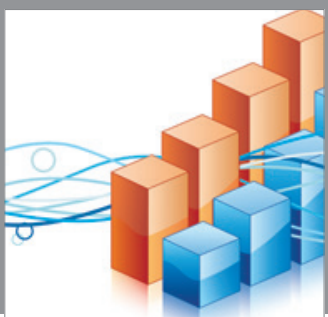

Advances in

Operations Research

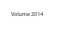

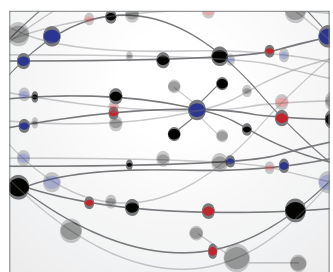

\section{The Scientific} World Journal
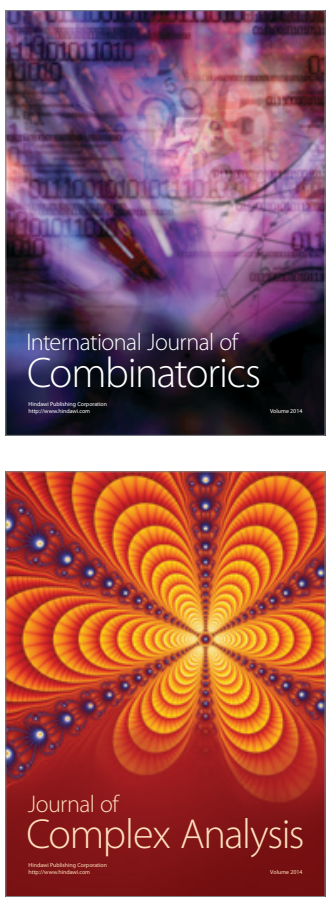

International Journal of

Mathematics and

Mathematical

Sciences
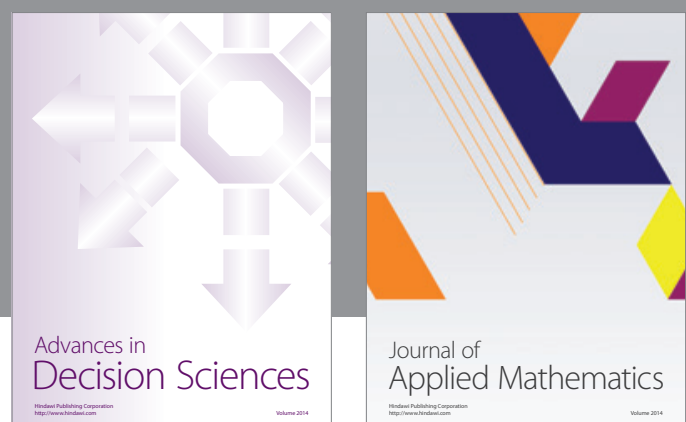

Journal of

Applied Mathematics
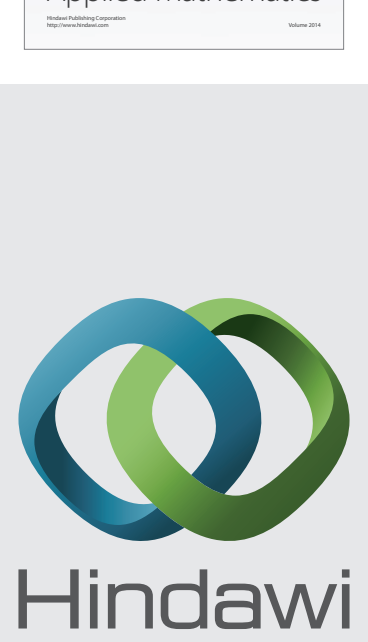

Submit your manuscripts at http://www.hindawi.com
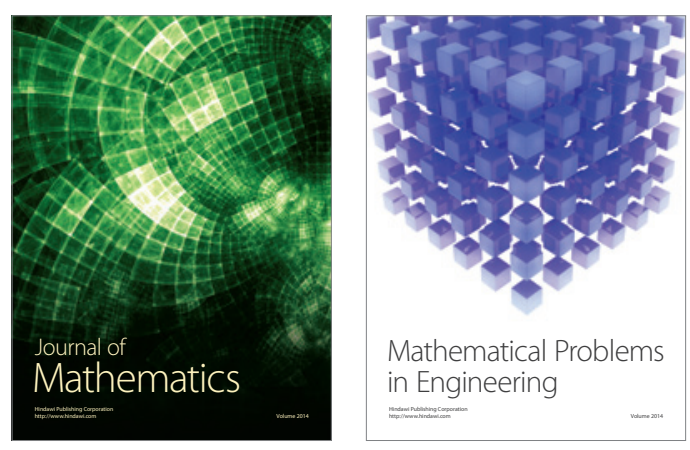

Mathematical Problems in Engineering
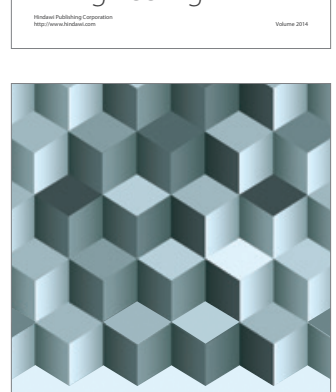

Journal of

Function Spaces
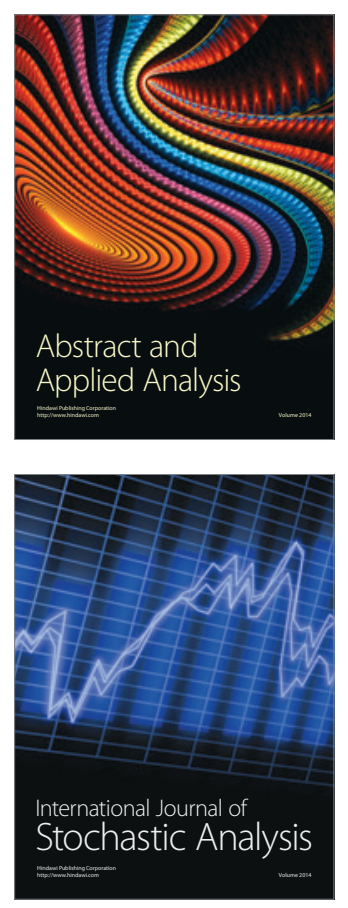

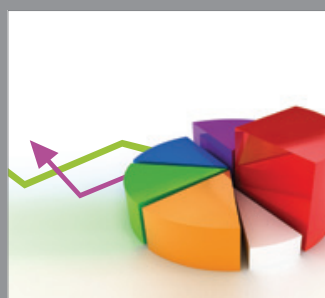

ournal of

Probability and Statistics

Promensencen
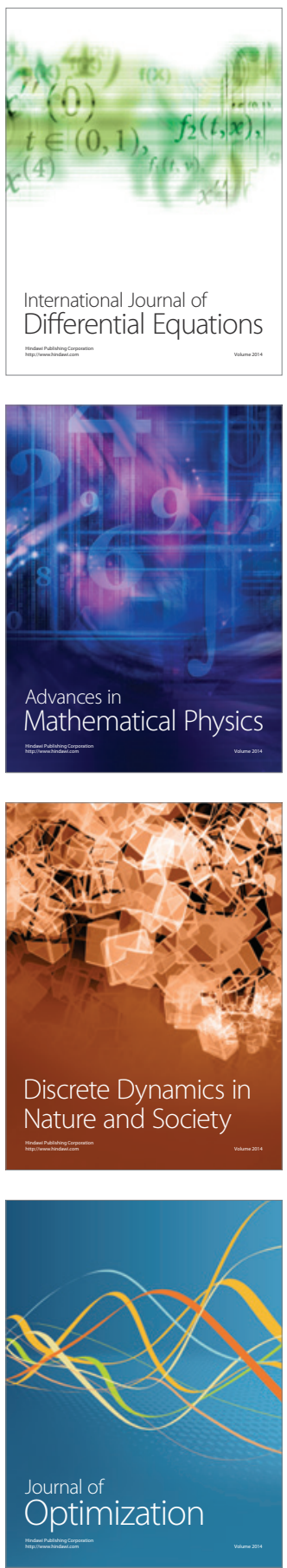